www.ijpsonline.com

\title{
Observation of Nursing Effect for Patients with Primary Liver Cancer Before and After Transcatheter Arterial Embolization
}

\author{
LAN LUAN AND LIN WANG ${ }^{*}$ \\ Department of Nursing, Jiangsu College of Nursing, Huai'an, 223005, 'Department of General Surgery, The Second People's \\ Hospital of Huai'an (The Affiliated Huai'an Hospital of Xuzhou Medical University), Huai'an, 223002, China
}

Luan and Wang: Nursing Effect for Patients with Primary Liver Cancer

To observe and analyze the nursing effect of primary liver cancer before and after transcatheter arterial embolization, 200 patients with advanced primary liver cancer who had been treated in the Second People's Hospital of Huai' an were selected as research subjects. All of them were subjected to transcatheter arterial embolization. During perioperative period, nursing was carried out for patients and they were explained about the method, purpose, results and cautions about the treatment; moreover, psychological nursing intervention, dietary intervention, auxiliary examination were performed. The postoperative puncture site and operative limb situations were observed and recorded for medication guidance. In this study, all patients were well treated. Adverse reactions after treatment mainly included liver area pain, digestive tract reaction, fever, which were relatively mild and effectively improved with timely medical measures with no severe complications being left. Comparison of quality of life score and mental status score before and after nursing showed significant difference. To guarantee a smooth and successful implementation of transcatheter arterial embolization for patients with primary liver cancer, the key is to develop a scientific and comprehensive nursing mode to improve curative effect 
Clinically, primary liver cancer is a common malignant tumor. With the change of life style, environment and dietary structure, the number of patients with primary liver cancer has been on a rise in recent years. Currently, the most common treatment for liver cancer is surgical resection. However, as most cases of primary liver cancer are in advanced stage, the recurrence rate after surgery is high and as some patients are accompanied with liver cirrhosis, the resection rate of early liver cancer is only $20-30 \%$ and most cases needed to be treatment with non-surgical regimens ${ }^{[1-3]}$. At present, existing literature on the etiology and pathogenesis of primary liver cancer did not yield a unified conclusion. It is well recognized that the primary liver cancer is associated with liver cirrhosis (fig. 1), viral hepatitis (fig. 2), aflatoxin (fig. 3) and environment factors. For patients who have missed the best opportunity for surgical resection or have no surgical indications, the main treatment approach is transcatheter arterial embolization, which can prolong the survival time on one hand and on the other restore opportunity for surgical resection for at least some patients. During the transcatheter arterial embolization, scientific nursing care is of vital importance. In this study, the nursing effect on primary liver cancer patients before and after

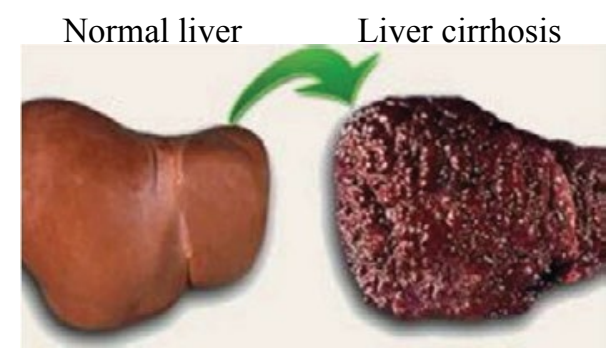

Fig. 1: Liver cirrhosis

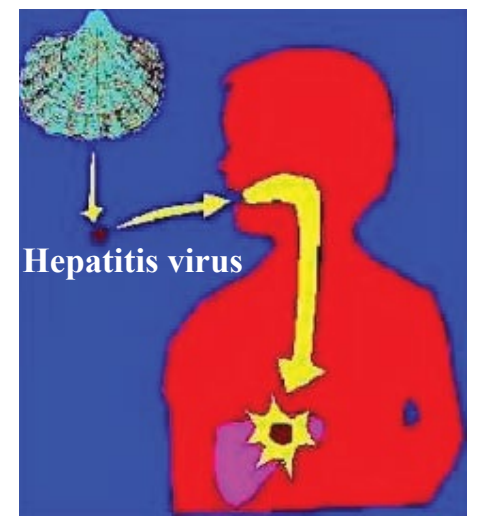

Fig. 2: Viral hepatitis
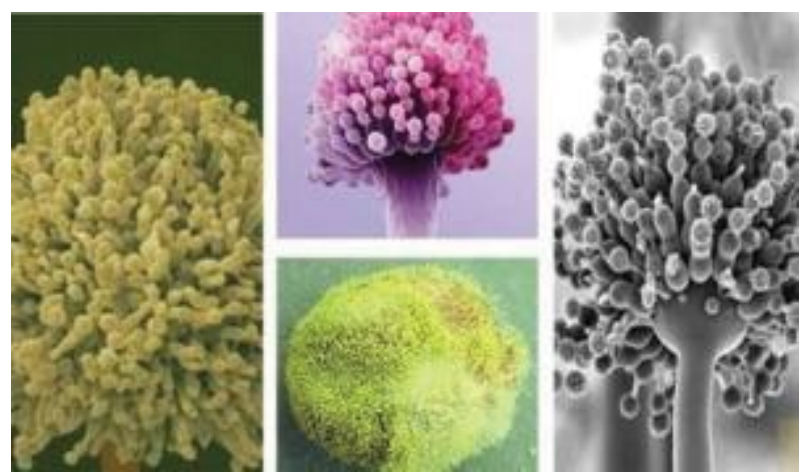

Fig. 3: Aflatoxin

transcatheter arterial embolization was investigated, which would provide valuable evidence for practical nursing. Two hundred patients with primary liver cancer who had been treated in the Second People's Hospital of Huai'an during May 2014 to March 2018 were selected as research subjects. The inclusion criteria included those patients meeting the clinical diagnosis and staging criteria of primary liver cancer prescribed by Chinese Society of Liver Cancer, Chinese anticancer Association, who were subjected to clinical and imaging examination, such as ultrasonic B, CT, MRI, hepatic arteriography, PET, serum alpha-fetoprotein determination ${ }^{[4,5]}$ and those with no contraindication to surgical treatment. The selected patients enjoyed the right to know and signed informed consent form. Those patients who were given other antitumor treatments within the past one month, those patients with severe heart, liver and kidney dysfunction, those patients with jaundice, ascites, cachexy and multi-site metastases and those patients with poor compliance were excluded. Of the 200 selected patients, there were 122 males and 78 females, 30-75 years old, with an average age of $50.6 \pm 3.2 \mathrm{y}$. Before treatment, intramuscular injection of $10 \mathrm{mg}$ of diazepam was given along with surgical site disinfection and local infiltration anesthesia. Puncture intubation was done via arteria cruralis beat area of right groin according to Seldinger technique. Under the Xray scan or CT guidance, the tumor site was clearly determined to which the catheter was extended ${ }^{[6]}$. The feeding artery of tumor was found into which the chemotherapeutic drugs were injected. Used interventional therapy drugs for liver cancer included cisplatin, hydroxyl glycyrrhizin, epirubicin, mitomycin, Brucea jananica oil injection, arsenic trioxide injection 
and elemene, $40 \%$ iodipin. According to the actual disease condition, the combined application of 2 or 3 drugs accompanied with iodipin was injected. Nursing method employed was preoperative basic nursing, which included establishing a harmonic patient-doctor relationship, making patients aware of ward environment, keeping the ward clean, making patients feel safe and comfortable. Preoperative dietary nursing such as instructing patients to take semi-liquid diet 1 or 2 days before operation, the diet should be light and easy to digest, prohibiting patients from drinking water for about $5 \mathrm{~h}$. Preoperative mental nursing was employed to establish a sound communication with patients to understand their moods, making targeted measures to eliminate anxiety, tension, anxiety, panic and depression of patients, imparting patients the knowledge of their disease and then letting them know the purpose, method, effect and cautions of the treatment, so as to remove the fear to improve their confidence and compliance. The postoperative nursing included closely focusing patients' disease conditions and monitoring their vital signs including respiratory rate, blood pressure, pulse, heart rate, body temperature; forcing the patients to take strict bed rest for $24 \mathrm{~h}$, any abdominal pain or peritoneal irritation should be informed to doctor in time. Particular nursing care should be given to puncture site and affected limb. The postoperative puncture site needs to be bound up for 12 $\mathrm{h}$, followed by bed rest for $24 \mathrm{~h}$ to prevent puncture site bleeding and hematoma. Keeping the right leg immobilized for $8 \mathrm{~h}$, examining the skin temperature, pallor and massaging the lower limb to prevent thrombosis; monitoring body temperature and instructing patients to drink adequate water, physical cooling or drug cooling should be employed in time if the body temperature is over $39^{\circ}$. Observing the gastrointestinal reaction since higher doses of chemotherapy drugs might stimulate gastrointestinal toxicity, embolization agents may backrush to peripheral artery of the duodenum, which would stimulate gastrointestinal mucosa and precipitate acute gastrointestinal reaction, thus resulting in nausea and vomiting. Therefore, the patients should be instructed to take light diet and if emesis occurs, patient's head should be titled to one side to prevent aspiration, coughing and choking. Performing finger pressing or acupuncturing at Zusanli, Neiguan to eliminate digestive tract symptoms ${ }^{[7,8]}$. Good nursing care should be provided to liver and kidney function; fluid infusion should be applied regularly before operation; recording urine output and urine color after surgery and maintaining urine output around $2500 \mathrm{ml}$. Finally, provision of rehabilitation guidance. In the phase of postoperative rehabilitation, mental nursing should be strengthened to increase patients' belief of rehabilitation belief to make them maintain positive $\operatorname{mood}^{[9]}$. In addition, instructing patients to perform appropriate exercises to improve immunity and teaching them heath knowledge via rehabilitation-propaganda to prevent postoperative complications. Moreover, making sure patients take medication, diet, rest and reexamination strictly as prescribed ${ }^{[10-15]}$. The adverse reactions were recorded. The mental status (anxiety scores, depression scores) and quality of life before and after nursing were compared. SPSS21.0 software was used for statistical analysis. The measured data was expressed in the form of mean \pm standard deviation and intergroup difference was compared using the $t$ test. The enumeration data was expressed by natural number (n) and percentage $(\%)$, and the intergroup difference was tested by $\chi^{2}$. The difference was of statistical significance when $P<0.05$. As shown in Table 1, the adverse reactions mainly included liver area pain, digestive tract reaction, fever, which were relatively mild and effectively improved with timely medical measures, without causing severe complications, such as puncture site bleeding, hematoma, deep venous thrombosis, etc. As shown in Table 2, the quality of life score after nursing is significantly higher than that before nursing, $P<0.05$. As shown in Table 3, the self-rating anxiety scale (SAS) and self-rating depression scale (SDS) scores after nursing are significantly higher than that before nursing, $P<0.05$. Early detection and early treatment are the key factors influencing the curative effect of primary liver cancer. Currently, the 5-y survival rate of primary liver cancer after surgery is about $70 \%{ }^{[16]}$, which is relatively satisfactory. So, patients should not lose confidence and accept treatment as soon as possible if being diagnosed with primary liver cancer. One of main measures to treatment advanced primary liver cancer is transcatheter arterial embolization. Drugs are injected into the liver tumor via catheter, which can greatly increase the concentration of chemotherapeutics around the liver tumor and thus exert good cytotoxic effect on the liver tumor cells. In addition, adverse reactions caused by intravenous chemotherapy could be prevented. A scientific nursing approach is the key to guarantee

\section{TABLE 1: ADVERSE REACTIONS}

\begin{tabular}{lcccc}
\hline $\begin{array}{l}\text { Number } \\
\text { of cases }\end{array}$ & $\begin{array}{c}\text { digestive } \\
\text { tract } \\
\text { reaction }\end{array}$ & $\begin{array}{c}\text { liver area } \\
\text { pain }\end{array}$ & fever & $\begin{array}{c}\text { Total } \\
\text { number }\end{array}$ \\
\hline 200 & 40 & 22 & 25 & 87 \\
\hline
\end{tabular}


www.ijpsonline.com

TABLE 2: COMPARISON OF QUALITY OF LIFE SCORE BEFORE AND AFTER NURSING

\begin{tabular}{lccccc}
\hline Time & Number of cases & $\begin{array}{c}\text { Physiological } \\
\text { function }\end{array}$ & Mental function & Social function & $\begin{array}{c}\text { Total quality of } \\
\text { life score }\end{array}$ \\
\hline After nursing & 200 & $0.97 \pm 0.32$ & $1.30 \pm 0.52$ & $1.29 \pm 0.15$ & $4.81 \pm 0.55$ \\
Before nursing & 200 & $0.43 \pm 0.29$ & $1.01 \pm 0.05$ & $0.82 \pm 0.23$ & $3.26 \pm 1.20$ \\
$t$ & & 3.58 & 5.31 & 5.09 & 8.36 \\
$\mathrm{P}$ & $<0.05$ & $<0.05$ & $<0.05$ & $<0.05$ \\
\hline
\end{tabular}

TABLE 3: COMPARISON OF SAS AND SDS SCORES BEFORE AND AFTER NURSING

\begin{tabular}{lccc}
\hline Time & $\begin{array}{c}\text { Number of } \\
\text { cases }\end{array}$ & SAS score & SDS score \\
\hline After nursing & 200 & $41.70 \pm 4.25$ & $40.61 \pm 2.80$ \\
Before & 200 & $48.92 \pm 5.05$ & $48.65 \pm 3.86$ \\
nursing & & 9.72 & 6.51 \\
$\mathrm{t}$ & & $<0.05$ & $<0.05$ \\
$\mathrm{P}$ & &
\end{tabular}

SAS is self-rating anxiety scale and SDS is self-rating depression scale

smooth implementation of transcatheter arterial embolization. Therefore, the preoperative preparation work needed be improved and real-time monitoring of vital signs of patients to be done to prevent emergency situations. Standard nursing operations should be carried out to prevent complications, increase patients' quality of life and accelerate their rehabilitation. In conclusion, to guarantee smooth implementation of transcatheter arterial embolization for patients with primary liver cancer, scientific and comprehensive nursing mode should be developed to improve curative effect. This nursing mode could prolong survival time and improve quality of life of patients, which is of great application value.

\section{REFERENCES}

1. Zhang LY, Wang YZ. Observation of nursing effect before and after transcatheter arterial embolization for patients with primary liver cancer. J Tradit Chin Med 2018;34(1):110-23.

2. Li H, Lu Q, Yang P, Liu WR, Wang HY, Li SM. Symptoms of patients accepting primary liver cancer surgery and their needs for continuing care. Chin J Nurs 2015;50(06):684-88.

3. Ma HL. Related factors of causing acute upper gastrointestinal hemorrhage after transcatheter arterial embolization for patients with primary liver cancer and nursing mode. World Latest Medicine Information 2015;15(43):69-70.

4. Nouso K, Miyahara K, Uchida D. Effect of hepatic arterial infusion chemotherapy of 5-fluorouracil and cisplatin for advanced hepatocellular carcinoma in the nationwide survey of primary liver cancer in Japan. Br J Cancer 2016;109(7):19047.

5. Asayama Y, Yoshimitsu K, Irie H. Poorly versus moderately differentiated hepatocellular carcinoma: vascularity assessment by computed tomographic hepatic angiography in correlation with histologically counted number of unpaired arteries. J Comput Assist Tomogr 2017;31(2):188-92.

6. Han YH. Analysis of reasons of fever occurring after implementing transcatheter arterial chemo embolization for patients with primary liver cancer and relevent nursing mode analysis. World Latest Medicine Information 2016;16(64):28891.

7. Jin X, Fangfang, Yu JX. Research progress on nursing for vomiting after implementating transcatheter arterial chemoembolization for patients with primary liver cancer. Shanghai Nursing Journal 2017;17(6):62-5.

8. Zhang Y, Zhao J, Guo D. Evaluation of short-term response of high intensity focused ultrasound ablation for primary hepatic carcinoma: utility of contrast-enhanced MRI and diffusionweighted imaging. Eur J Radiol 2016;79(3):347-52.

9. Gong L, Shi L, Sun J. Comparative survival analysis of adjuvant therapy with iodine-131-labeled lipiodol to hepatic resection of primary hepatocellular carcinoma: A metaanalysis. Nucl Med Commun 2017;35(5):484-92.

10. Gao W, Wang Y, Basavanagoud B, Jamil MK. Characteristics Studies of Molecular Structures in Drugs. Saudi Pharm J 2017;25(4):580-6.

11. Ge S, Wang L, Liu Z, Jiang S, Yang X, Yang W, et al. Properties of Nonvolatile and Antibacterial Bioboard Produced from Bamboo Macromolecules by Hot Pressing. Saudi J Biol Sci 2018;25(3):474-8.

12. Alkowni R, Jodeh S, Hussein F, Jaradat N. Phytochemical Screening and Antibacterial Activity of Cyclamen Persicum Mill Tuber Extracts. Pak J Pharm Sci 2018;31(1):187-92.

13. Al-Enazi NM, Awaad AS, Zain ME, Alqasoumi SI. Antimicrobial, Antioxidant and Anticancer Activities of Laurencia Catarinensis, Laurencia Majuscula and Padina Pavonica Extracts. Saudi Pharm J 2018; 26(1):44-52.

14. Afsar CU, Karabulut M, Karabulut S, Alis H, Binboga $\mathrm{S}$, Bilgin E, et al. Circulating Monocyte Chemoattractant Protein-1 (Mcp-1/Ccl-2) as a Potential Biomarker for the Diagnosis of Pancreatic Adenocarcinoma. Acta Medica Mediterr 2017;33(3):501-7.

15. Ai F, Wang J, Li Y, Ma Y. Effect of Drug Particle Size on Complexation, Physicochemical Properties and Dissolution of Cyclodextrin Inclusion Complexes. Indian J Pharm Sci 2017;79(1):131-8.

16. Dong $\mathrm{X}$, Jin $\mathrm{K}, \mathrm{Hu} \mathrm{X}$. Antitumor effect of FP3 in combination with cetuximab on patient-derived tumor tissue xenograft models of primary colon carcinoma and related lymphatic and hepatic metastases. Int J Mol Med 2016;30(1):126-132.

This is an open access article distributed under the terms of the Creative Commons Attribution-NonCommercial-ShareAlike 3.0 License, which allows others to remix, tweak, and build upon the work non-commercially, as long as the author is credited and the new creations are licensed under the identical terms

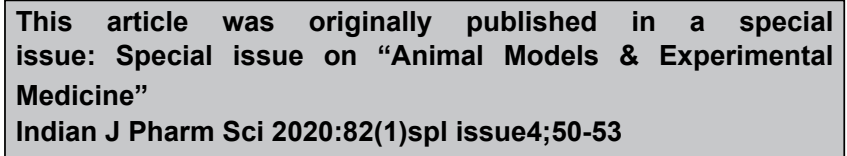

